PROGNOSIS OF INFANTILE SPASMS (WEST SYNDROME)

Investigators at the Instituto di Neuropsychiatria Infantile, University of Rome, Italy, followed 58 cases of West syndrome for 3 years or more (mean 5 yr 5 mo). Eight (14\%) were classified as idiopathic, and 50 (86\%) were symptomatic, of which 17 were secondary to birth asphyxia or cerebral hemorrhage and 11 ( $19 \%$ of total) had tuberous sclerosis. All were treated with АСТн, 25-50 IU daily for 3 weeks and 25 IU daily for further 3 weeks or more if no response.

IQ was normal or borderline (>70) in 5 (8\%) and retarded in $53(92 \%)$. A low IQ at final follow-up was correlated with developmental and/or neurological abnormalities before the onset of spasms, symptomatic etiology, and abnormal CT findings (cerebral atrophy 30 (52\%), calcifications 11 (19\%), callosal agenesis $3(5 \%))$. An IQ above 50 in 13 (23\%) was associated with early speech development (first word before 2 years and two-word sentence before 3 years) and an IQ below 50 was found in children with retarded speech development. Motor milestones did not correlate with subsequent mental development even in cases without neuromotor impairment. Whereas clinical and CT evidence of cerebral damage correlated with mental retardation, the absence of cerebral abnormalities was not predictive of a normal mental development. A poor prognosis in ws was predictable but normal development at follow-up was not reliably associated with favorable variables at onset. (Favata I, Leuzzi Y, Curatolo P. Mental outcome in west syndrome: prognostic value of some clinical factors. J Ment Def Res 1987; 31: 9-15).

COMMENT: Of 1,558 cases of West syndrome reported in the 1 iterature between 1954 and 1973, a 20-year span, 624 (40\%) were idiopathic and 934 (60\%) were symptomatic (Lacy JR, Penry J. Infantile Spasms. Raven Press, New York, 1976). The unusually high percentage of symptomatic cases in this study could explain the relatively higher incidence of mental retardation. Could the $52 \%$ incidence of cerebral atrophy demonstrated by CT be caused in part by ACTH? As the authors point out, their data related to a poor mental outcome cannot strictly be regarded as prognostic factors since they are an expression of the symptomatic character of WS, accounting for $86 \%$ of their cases. Among 11 patients with tuberous sclerosis, 4 (36\%) had an IQ $>50$ at follow-up and 7 $(64 \%)$ were moderately to severely retarded. Unfortunately, the relation of IQ to the age at time of treatment and response to ACTH was not evaluated, a frequently debated question. In a study at the Mayo Clinic (Millichap JG, Bickford RG. JAMA 1962; 182:523), psychomotor retardation was generally more severe in patients with symptomatic infantile spasms than in those with cryptogenic seizures. ACTH was possibly more effective in patients with IQ's of 70 or above than in those with retarded development before treatment. Control of infantile spasms and hypsarrhythmia by ACTH was approximately $50 \%$ and equal in cryptogenic and symptomatic groups of patients.

\title{
TOXICITY OF NITRAZEPAM (MOGADON)
}

A pediatric neurologist and his associates at the children's Mercy Hospital, Kansas City, report 6 deaths among 80 patients with intractable epilepsy treated with nitrazepam. The patients who died were 13-39 mos of age (mean 28 mos) and had received nitrazepam for 2 to 19 months (mean 8 mos) in a dosage of 0.9-2.7 $\mathrm{mg} / \mathrm{kg} / \mathrm{d}$ (mean $\mathrm{l} .4$ ). Up to 2 additional but unnamed antiepileptic 
drugs were used concurrently but no patient received other benzodiazepines. The dosage of nitrazepam in the 6 fatal cases was significantly higher and approximately double that in 22 survivors of the same age range (mean $0.7 \mathrm{mg} / \mathrm{kg} / \mathrm{d}$ ). Patients in both groups had multiple seizures, including generalized tonicclonic, myoclonic and focal types but none had infantile spasms or absence attacks. Perinatal asphyxia, Prader-Willi syndrome, pertussis vaccine-related encephalopathy, and nonketotic hyperglycinemia were the etiological diagnoses in 4 of the 6 fatal cases. Three of the 6 had known factors contributing to death: congestive heart failure, aspiration of gastric contents, and unexplained hyperthermia, shock, and respiratory failure. Three patients died unexpectedly and autopsies in 2 were unrevealing. The cause of death was undertermined but a nitrazepam-induced swallowing disturbance and aspiration was suspected. The authors recommend that the use of nitrazepam in young children should be restricted to those resistant to other antiepileptic drugs, the dose should not exceed $0.8 \mathrm{mg} / \mathrm{kg} / \mathrm{d}$, and children with prior swallowing difficulties should be observed closely. (Murphy JV et al. Deaths in young children receiving nitrazepam J Pediat 1987; 111:145-147).

COMMENT: Nitrazepam (Mogadon) is considered the most effective benzodiazepine for control of infantile spasms and other myoclonic seizures. It is not usually recommended for the treatment of generalized tonic-clonic seizures, as employed in this study, which might explain the necessity for the larger doses in the affected infants.

In a study at Children's Memorial Hospital, Chicago (Millichap JG, Ortiz WR. Am J Dis Child 1966; 112: 242), the side effects of nitrazepam in a trial involving 36 infants and children with myoclonic seizures included drowsiness (50\%), ataxia (20\%), hypotonia (20\%), and muscular weakness (9\%). Anorexia and vomiting developed in $2 \%$ and skin rash in $2 \%$. the most serious adverse effects of nitrazepam in our study were symptoms suggesting autonomic dysfunction, previously unreported. Excessive drooling of saliva occurred in $9(25 \%)$ infants and pulmonary congestion with wheezing developed in 4 ( $11 \%$ ) debilitated infants with diplegia and severe retardation. Miosis of the pupils was also noted. The pulmonary symptoms required withdrawal of the drug or reduction of the dose to less effective levels and possible fatalities were avoided. The nitrazepam-induced drooling and aspiration in some cases have recently been explained by a delay of cricopharnygeal relaxation (Wyllie $\mathrm{E}$ et al. N Engl J Med 1986; 314:35-38). The tolerance that develops to nitrazepan and the autonomic and sedative side-effects of larger doses seriously detract from the usefulness of this agent in long-term theraphy of infantile spasms. In debilitated infants, perhaps nitrazepam should be contraindicated.

\section{WITHDRAWAL OF ANTIEPILEPTIC TREATMENT}

Investigators at the Division of Neurology and Clinical Neurophysiology, Hospital General de Catalunya, Barcelona, Spain, evaluated the withdrawal of antiepileptic treatment over a one year period in 608 epileptics who had been seizure-free for 5 years. Relapses occurred in 144 (23.7\%) of the total group. Among 474 patients in whom details of seizure types were available, relapses occurred in 119 (25.1\%) of the total. Idiopathic generalized epilepsies showed a $19.6 \%$ relapse reate whereas symptomatic generalized epilepsies relapsed in $37.9 \%$ of patients. The lowest relapse rate occurred in patients with absence seizures $(9.9 \%)$ and the highest rates in those with west 